\title{
Sistema de Baixo Custo para Nucleação de Pré-Trincas por Fadiga em Corpos de Prova de Tenacidade à Fratura
}

\author{
V.A. Guimarães, L.R.O. Hein, J. Zangrandi Filho \\ Departamento de Materiais e Tecnologia, FEG, UNESP, \\ 12500-000 Guaratinguetá-SP, Brazil
}

Received: December 13, 2000; Revised: June 5, 2001

\begin{abstract}
Fracture toughness testing becomes a very important parameter for mechanical design and maintenance of parts and structures. This work presents the design and construction of a machine device to perform the pre-crack nucleation on $\mathrm{SE}(\mathrm{B})$ specimens for fracture toughness testing. Employing this device, it is possible to test materials for $\mathrm{K}_{\mathrm{Ic}}, \mathrm{CTOD}$ and $\mathrm{J}_{\mathrm{Ic}}$ in a conventional tensile test equipment, assisted by a data acquisition system. The device is based on the concept of loading from an eccentric mechanism, controlling the displacement by means of a marking ring. The main contribution of this work is the low cost technology offered for laboratories, without high priced servo-hydraulic testing machines, giving them autonomy for the execution of fracture toughness tests. The experimental results satisfied the expectation about crack nucleation on $\mathrm{SE}(\mathrm{B})$ and applied loading control.
\end{abstract}

Keywords: fracture toughness, fracture mechanic, mechanical testing

\section{Revisão Bibliográfica}

Um dos maiores problemas atuais no acompanhamento da integridade de componentes mecânicos é a possibilidade de ocorrência de uma fratura frágil, repentina e inesperada, mesmo quando o componente esteja submetido a condições normais de operação. O dano que pode ocorrer em uma estrutura, principalmente se houver a presença de um concentrador de tensão, vai depender, basicamente, da capacidade do material absorver deformações plásticas localizadas, gerando uma região deformada na ponta da trinca $^{1,2}$. A presença de um entalhe em um componente mecânico cria regiões nas proximidades da ponta da trinca com estados triaxiais de tensões, o que restringe a deformação plástica, ou seja, tende a fragilizar o material. A diminuição da temperatura do ensaio ou o aumento da taxa de deformação tende a diminuir a ductilidade, provocando uma redução na tenacidade à fratura.

A tenacidade à fratura pode ser avaliada através de diversos parâmetros. Entre os parâmetros mais comuns, para avaliação da tenacidade, destacam-se a medida de energia absorvida em um ensaio de impacto e a temperatura de transição. Mais recentemente, foram desenvolvidos os parâmetros de projeto mecânico ligados à mecânica da fratura, que são realizados em corpos de prova com uma trinca aguda na raiz do entalhe que garante, dentro de certos

e-mail: valdir@feg.unesp.br

Trabalho apresentado no $14^{\circ}$ CBECIMAT, Águas de São Pedro, Dezembro de 2000 . limites, a máxima severidade possível de tensões para o entalhe $^{3}$. Esses ensaios são: tenacidade à fratura em deformação plana $\left(\mathrm{K}_{\mathrm{IC}}\right)$, deslocamento de abertura da ponta da trinca crítico (CTOD) e o parâmetro crítico em termos da integral $\mathrm{J}\left(\mathrm{J}_{\mathrm{IC}}\right)$. Os ensaios de tenacidade à fratura do tipo $\mathrm{K}_{\mathrm{IC}}$ são mais indicados para materiais frágeis de elevada resistência mecânica. Já os ensaios CTOD e integral $\mathrm{J}_{\mathrm{IC}}$ permitem estabelecer parâmetros de tenacidade à fratura para materiais dúteis de menor resistência mecânica, em condições elasto-plásticas de deformação.

\subsection{Ensaio $K_{I C}$}

O ensaio $\mathrm{K}_{\mathrm{IC}}$ é empregado para materiais frágeis em que a condição de comportamento linear elástico até a ruptura é valido. As principais normas técnicas que tratam dos ensaios K KC são a ASTM E 399-90 e a BS 7448-1991 que normalizam conjuntamente as formas de obtenção do $\mathrm{K}_{\mathrm{IC}}$, $\mathrm{J}_{\mathrm{IC}}$ e do CTOD $^{5}$. A norma ASTM E 399-90 prevê para a obtenção do $\mathrm{K}_{\mathrm{IC}}$ diversos tipos de corpo de prova. Os corpos de prova mais utilizados são os do tipo $\mathrm{SE}(\mathrm{B})$ e o $\mathrm{C}(\mathrm{T})$. Esses dois corpos de prova se diferenciam basicamente pelo tipo de solicitação que são submetidos. O cdp do tipo $\mathrm{SE}(\mathrm{B})$ é solicitado por flexão em três pontos, enquanto o corpo de prova $\mathrm{C}(\mathrm{T})$ é solicitado por tração como mostra a Fig. 1. Todos os corpos de prova apresentam 


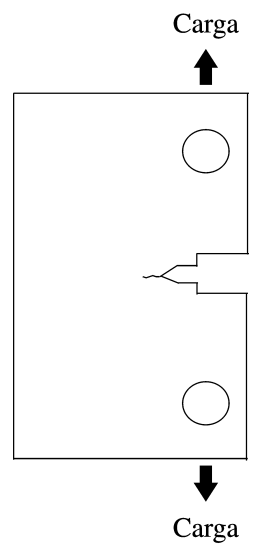

(a)

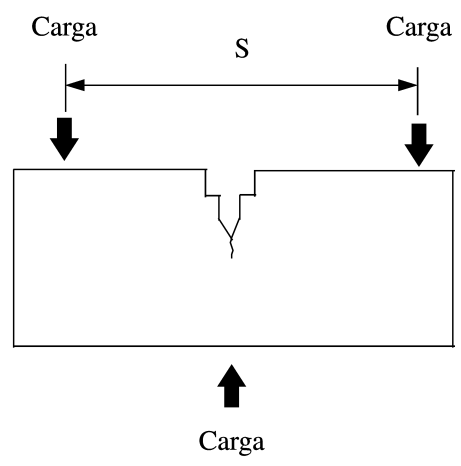

(b)
Figura 1. Esquema representativo da forma de aplicação de carregamento para os corpos de provas de tenacidade à fratura dos tipos: (a) C(T); (b) $\mathrm{SE}(\mathrm{B})$.

uma trinca aguda na raiz do entalhe obtida por fadiga. Esta trinca deve ser produzida dentro de determinados parâmetros de tamanho e de tensão máxima de acordo com a norma ASTM E 399-90.

\subsection{Ensaio JIC}

Semelhante ao $\mathrm{K}_{\mathrm{IC}}$, o ensaio $\mathrm{J}_{\mathrm{IC}}$ caracteriza a resistência à fratura, porém, é utilizado em condições elasto-plásticas onde ocorre uma propagação estável da trinca antes da fratura final. $\mathrm{O}$ processo de fratura em materiais dúcteis é precedido de um crescimento lento e estável da trinca. Durante o ensaio $\mathrm{J}_{\mathrm{IC}}$ a fratura se inicia a partir de uma pré-trinca aguda obtida por fadiga, que quando solicitada sofrerá, inicialmente, um processo de arredondamento de sua raiz e em seguida ocorre um crescimento estável da trinca. $\mathrm{O}$ valor critico de $\mathrm{J}$ que corresponde ao início de crescimento estável da trinca, em geral $2 \%$ da pré-trinca, é chamado de $\mathrm{J}_{\mathrm{IC}}{ }^{6}$.

Os corpos de prova utilizados para a determinação do $\mathrm{J}_{\mathrm{IC}}$ podem ser do tipo $\mathrm{SE}(\mathrm{B})$ ou $\mathrm{C}(\mathrm{T})$, porém a norma $\mathrm{ASTM}$ E 813-89 normaliza esses corpos de prova com algumas variações de geometrias em relação às amostras utilizadas para a obtenção do KIC. A obtenção de $\mathrm{J}_{\mathrm{IC}}$ é realizada através da obtenção experimental de uma curva de $\mathrm{J}$ em função da resistência ao crescimento da trinca ou Curva $\mathrm{R}^{7}$.

\subsection{Ensaio CTOD}

A maior parte dos aços de médio e baixo carbono que são utilizados em aplicações estruturais, normalmente são utilizados em espessuras que não são suficientes para manter as condições de deformação-plana nas temperaturas usuais de trabalho. Desse modo, em um número bastante grande de aplicações, os conceitos da mecânica da fratura linear elástica, não podem ser aplicados, devido ao comportamento elasto-plástico desses materiais. Em 1961, Weels ${ }^{8}$ propôs que o comportamento a fratura nas vizinhanças de uma trinca aguda, poderia ser caracterizado pelo deslocamento de abertura entre os planos da trinca - "crack- tip opening displacement" (CTOD). Posteriormente, Weels demonstrou que o conceito da abertura da ponta da trinca era análogo ao conceito descrito por Irwin ${ }^{9}$ em 1968, que relaciona a tenacidade à fratura em deformação plana, $\mathrm{K}_{\mathrm{IC}}$, com o valor do CTOD - $(\delta)$.

O parâmetro de tenacidade CTOD, é baseado na medida da abertura da ponta da trinca, e é obtido a partir da medida do deslocamento $\mathrm{V}_{\mathrm{p}}$ realizada por um “clip-gage” devidamente instalado na abertura do entalhe do corpo de prova. A conversão do deslocamento $\mathrm{V}_{\mathrm{p}}$ obtida pelo "clip-gage" para o valor do CTOD no ensaio SE(B) é realizada através da determinação de um centro de rotação do corpo de prova situado abaixo da trinca, ilustrado pela Fig. 2. Esse centro de rotação é gerado pelo carregamento aplicado à amostra, que provoca uma deformação em torno de um ponto denominado como centro aparente de rotação ${ }^{10,11}$. Através da análise da Fig. 2, pode-se verificar que o valor do CTOD ( $\delta$ ) pode ser facilmente determinado por semelhança de triângulos e calculado, a partir do deslocamento de abertura da boca da trinca Vp, pela Eq. (1).

$$
\frac{\delta}{V_{p}}=\frac{r_{p}\left(W-a_{0}\right)}{r_{p}\left(W-a_{0}\right)+a_{0}+z}
$$

Onde:

$\delta$ - Deslocamento de abertura da ponta da trinca;

$\mathrm{r}_{\mathrm{p}}$ - Fator de rotação plástica;

W - Largura do corpo de prova;

a 0 - Comprimento da trinca;

$\mathrm{V}_{\mathrm{p}}$ - Deslocamento de abertura da boca da trinca;

Z - Distância do ponto de medição do extensômetro em relação à face do corpo de prova.

Entre os ensaios utilizados para avaliar a tenacidade à fratura dos materiais, o ensaio CTOD é o que apresenta a

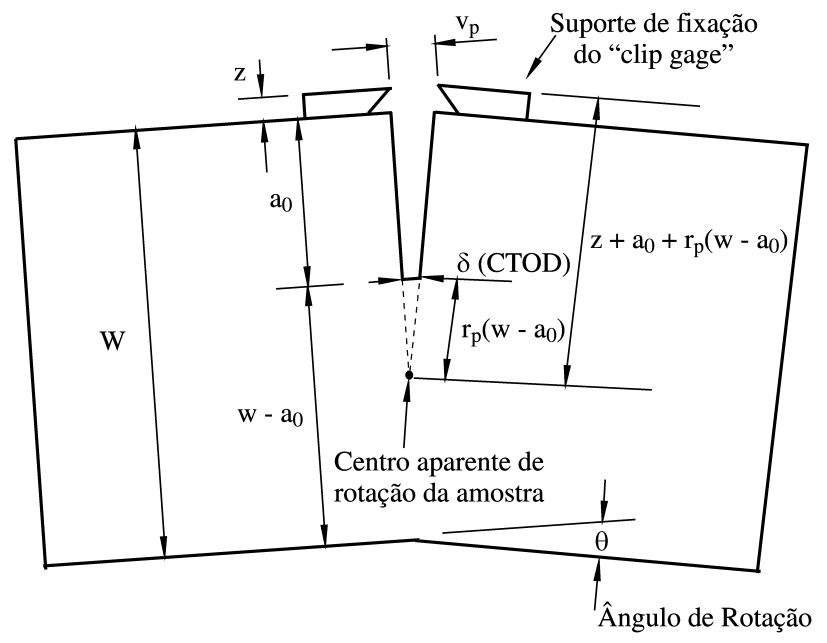

Figura 2. Representação esquemática da abertura da trinca durante um ensaio de flexão em um corpo de prova do tipo $\mathrm{SE}(\mathrm{B})^{10}$. 
maior simplicidade de execução. A principal vantagem da utilização do ensaio CTOD é devida à possibilidade da sua aplicação tanto em materiais de elevada resistência mecânica, onde predominam os conceitos da mecânica da fratura linear-elástica, quanto em materiais dúcteis onde se tem o comportamento elasto-plástico. Desse modo, o ensaio CTOD pode ser bastante indicado em pesquisa e desenvolvimento de novas microestruturas, onde não se tenha resultados prévios do comportamento de fratura dos materiais.

\section{Objetivos}

O presente trabalho tem como objetivos o projeto e fabricação de uma máquina para a nucleação de pré-trincas de fadiga em corpos de prova do tipo $\mathrm{SE}(\mathrm{B})$ para a realização de ensaios de tenacidade à fratura. Com este equipamento torna-se possível a realização dos ensaios do tipo $\mathrm{K}_{\mathrm{IC}}$, CTOD e $\mathrm{J}_{\mathrm{IC}}$, em uma máquina de tração simples convencional instrumentada por um sistema de aquisição de dados. A contribuição que este projeto proporciona é a criação de tecnologia para laboratórios, com pequena quantidade de recursos financeiros e que não dispõem de máquinas servo hidráulicas para ensaios dinâmicos, conseguirem autonomia para a execução de ensaios de tenacidade à fratura

\section{Metodologia}

Inicialmente foram realizadas as etapas de projeto e dimensionamento da parte estrutural, desenho e detalhamento de todos os seus componentes. Posteriormente foi realizada a montagem e os testes iniciais de funcionamento onde foram nucleadas trincas em diversos corpos de prova apropriados para a realização dos ensaios do tipo $\mathrm{K}_{\mathrm{IC}}$, CTOD e J $\mathrm{J}_{\mathrm{IC}} \mathrm{O}$ equipamento se baseia na concepção de aplicação de carga em um corpo de prova a partir de um mecanismo excêntrico onde se tem um controle de deslocamento por um anel graduado.

A máquina para nucleação de pré-trincas de fadiga é uma máquina de pequeno porte. A concepção do equipamento se baseia em um eixo com dois anéis excêntricos onde se pode regular um deslocamento dentro de uma faixa de 0 a $1 \mathrm{~mm}$ com precisão em torno de $0,05 \mathrm{~mm}$. Este deslocamento possibilitará a aplicação de cargas da ordem de até $600 \mathrm{kgf}$. $\mathrm{O}$ eixo excêntrico que tem como função aplicar movimentos alternados no corpo de prova transmite um esforço concentrado no entalhe, gerando uma trinca por fadiga no corpo de prova. Na parte superior da estrutura foi instalado um suporte para a fixação do corpo de prova. Este suporte inicialmente foi projetado para a fixação de corpos de prova do tipo SE(B). Posteriormente será desenvolvido um dispositivo para ensaios em corpos de prova do tipo $\mathrm{C}(\mathrm{T})$. A Fig. 3 mostra de forma esquemática os componen-

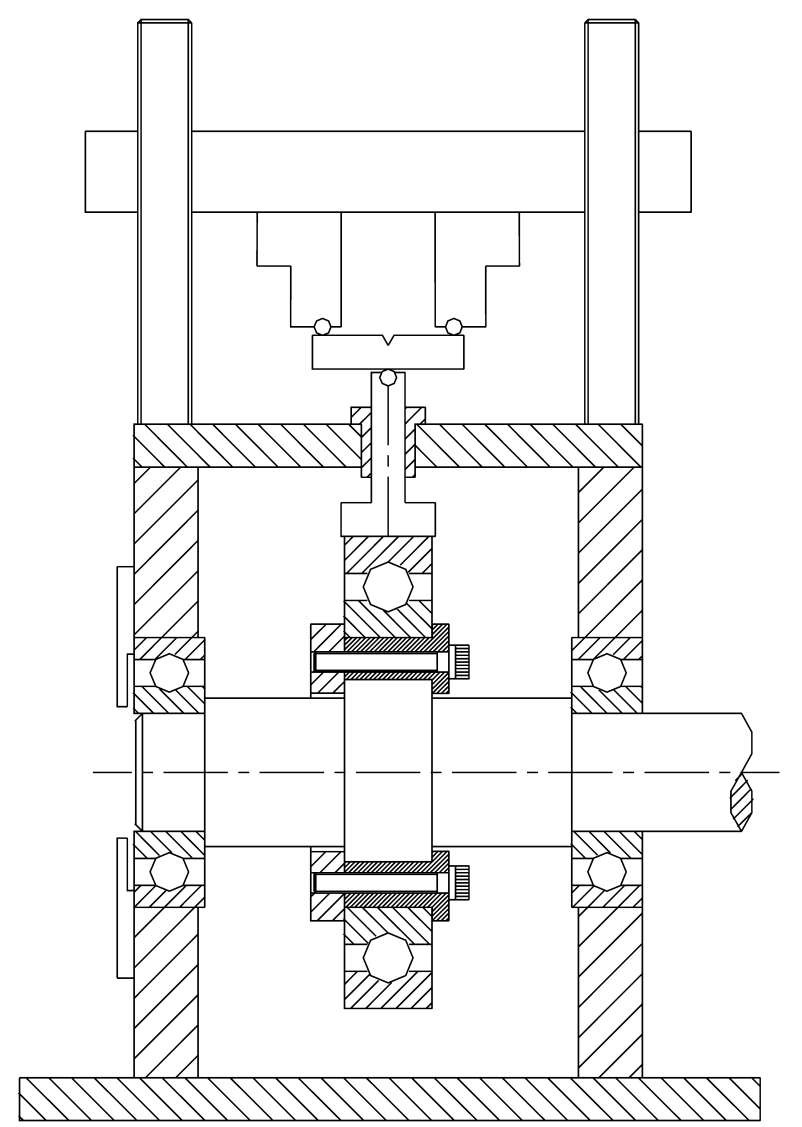

Figura 3. Representação esquemática da máquina de fadiga para nucleação de trincas em um corpo de prova do tipo $\mathrm{SE}(\mathrm{B})$.

tes onde pode-se verificar o sistema que será utilizado para ensaios em corpos de prova com fixação em três pontos.

Após a montagem do equipamento foram realizados diversos ensaios, sendo que o carregamento foi controlado através da utilização de uma instrumentação realizada com o auxílio de extensômetros colados no atuador. Dentro deste projeto também foram construídas as garras e sistemas de fixação da máquina de tração, apropriados para a realização de ensaios de mecânica da fratura.

\section{Resultados e Discussão}

O equipamento desenvolvido para a nucleação de trincas foi fabricado quase que na sua totalidade na oficina de apoio a pesquisa do Departamento de Materiais e Tecnologia. Uma das partes de maior dificuldade na fabricação foi a usinagem do eixo principal da máquina, onde o requisito principal foi a necessidade de um anel central com um diâmetro de $54 \mathrm{~mm}$ que estivesse com uma excentricidade de $0,35 \mathrm{~mm}$ em relação a linha de simetria do eixo. Toda a sua parte estrutural foi usinada a partir de placas de aço microligado, sendo que o eixo, anéis excêntricos e o dispositivo de fixação do corpo de prova foram usinados em aço 


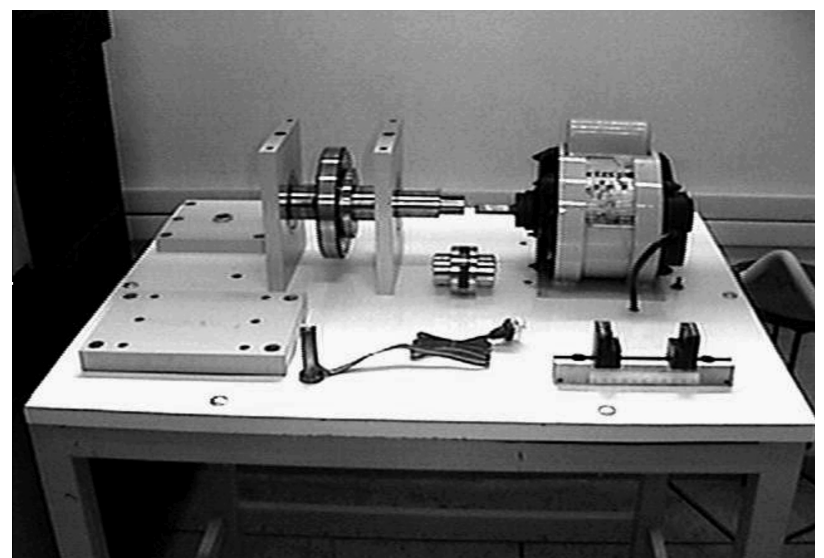

Figura 4. Principais componentes do projeto antes da montagem.

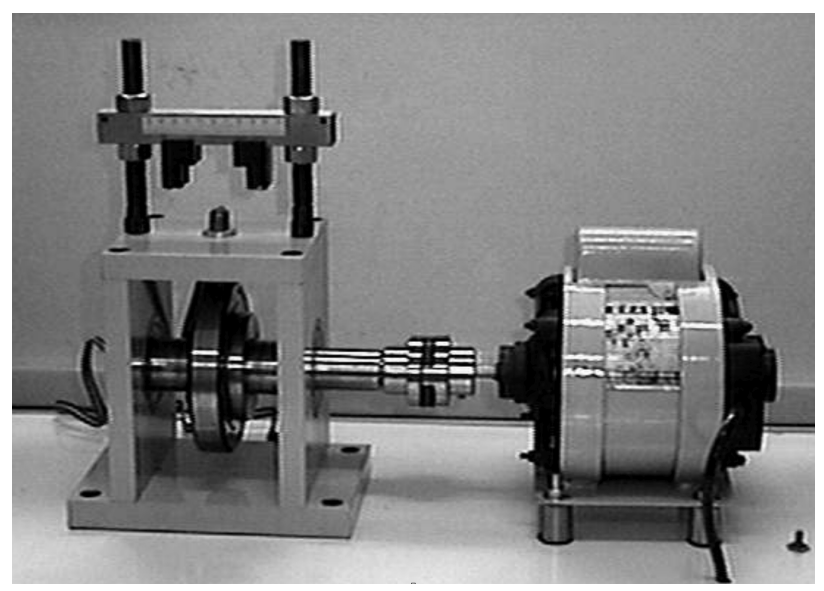

Figura 5. Equipamento para nucleação de trincas em corpos de prova do tipo flexão em três pontos $\mathrm{SE}(\mathrm{B}$.)

SAE 4140 e posteriormente temperados. A Fig. 4 mostra as partes principais do equipamento e a Fig. 5 mostra o equipamento montado com todos os seus componentes. A aplicação da carga é fixada por um controle de deslocamento, que não se altera durante todo o ensaio. No entanto, a carga aplicada que apresenta uma tendência de diminuição à medida que ocorre o crescimento da trinca pode ser controlada através do sistema de aquisição de dados. A instrumentação do atuador que aplica a carga no corpo de prova foi realizada através da colagem de dois "strain gage" na forma de roseta na sua base, saindo um conjunto de cabos apropriados para conexão no sistema de aquisição de dados como mostra a Fig. 6.

Antes da montagem do sistema foi realizada a calibração do atuador . Esta calibração foi feita colocando- se o pino em uma máquina convencional de ensaios mecânicos com uma célula de carga calibrada, onde aplicou-se um carregamento de compressão. Com este procedimento foi obtida uma curva de calibração mostrada pela Fig. 7, onde pode-se verificar que o coeficiente de correlação obtido, foi próximo a um para carregamentos aplicados até a ordem de 500 kgf. Através desta curva de calibração foi desenvolvido

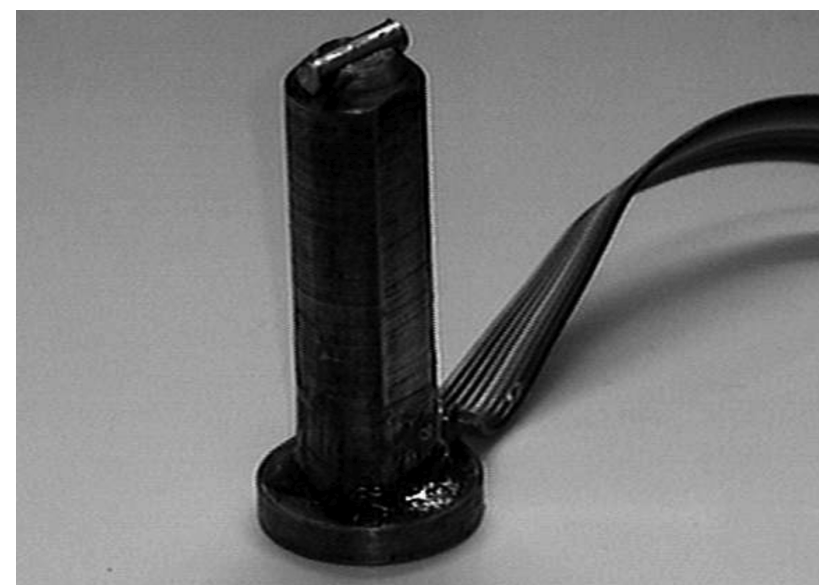

Figura 6. Atuador com sistema de cabos para conexão em sistema de aquisição de dados.

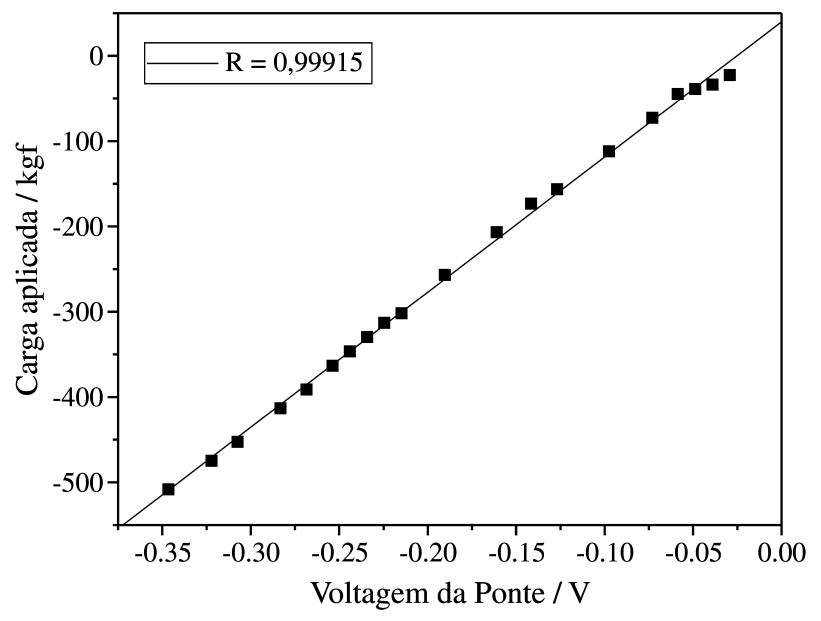

Figura 7. Curva de calibração do pino correlacionando a carga aplicada pela variação de voltagem no strain gage, onde obteve-se coeficiente de correlação de 0,99915 .

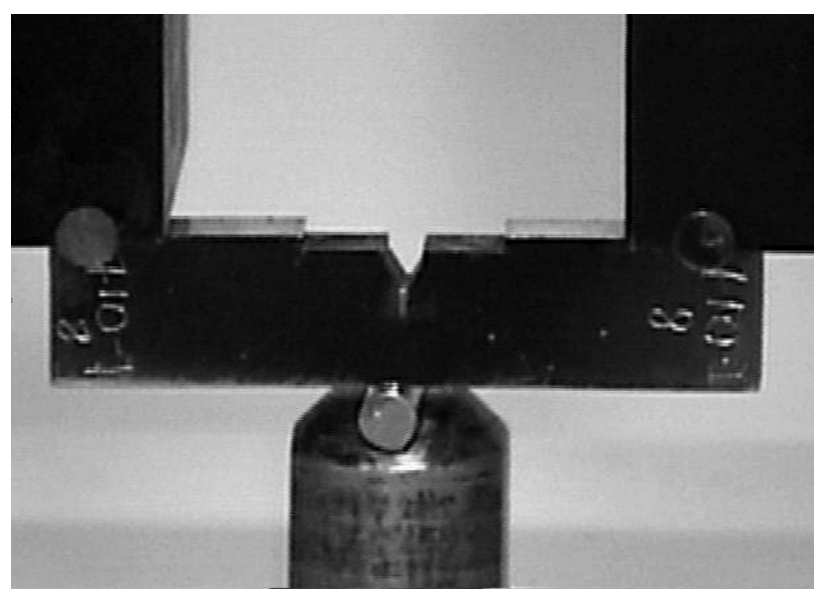

Figura 8. Trinca sendo nucleada em um corpo de prova de flexão em três pontos do tipo $\mathrm{SE}(\mathrm{B})$.

um programa no Software LabView, que possibilitará fazer as leituras dos carregamentos aplicados em função do 


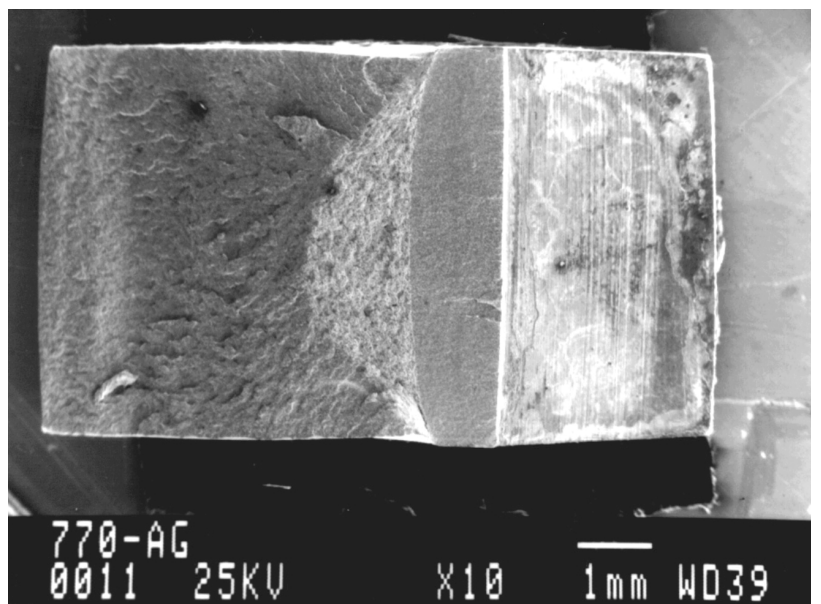

Figura 9. Superfície de fratura de um corpo de prova de flexão em três pontos do tipo $\mathrm{SE}(\mathrm{B})$ após o ensaio de CTOD.

tempo, permitindo a contagem do número de ciclos e o ajuste da carga.

Após a montagem do sistema foram realizados diversos ensaios em corpos de prova de flexão em três pontos. As trincas foram nucleadas de forma satisfatória, e atenderam os requisitos solicitados pelas normas no que se refere ao tamanho da trinca e ao carregamento aplicado. A Fig. 8 mostra uma trinca nucleada pelo dispositivo em um corpo de prova do tipo $\mathrm{SE}$ (B) e a Fig. 9 mostra a superfície de fratura do corpo de prova após um ensaio de CTOD onde é possível verificar a trinca obtida por fadiga, a região de crescimento estável da trinca e a região onde ocorreu a fratura final.

\section{Conclusões}

A geometria das trincas nucleadas pelo sistema atenderam os requisitos solicitados pelas normas da ASTM que se referem aos ensaios de tenacidade à fratura.

O controle dos carregamentos aplicados realizados pelo pino instrumentado foram precisos, podendo-se facilmente controlar os valores de carga máxima de fadiga previstos nas normas de tenacidade à fratura.

De forma geral, o sistema apresentou-se de forma eficiente, podendo ser utilizado como uma forma alternativa para a nucleação de pré-trincas por fadiga em corpos de prova de tenacidade à fratura.

\section{Agradecimentos}

Os autores agradecem a FUNDUNESP pelo suporte financeiro e o Prof. Juan Perez Ipina do Grupo de Mecánica de Fractura, da Facultad de Ingeniería da Universidad Nacional del Comahue - Argentina, pela auxílio inicial na concepção do projeto.

\section{Referências Bibliográficas}

1.Dieter, G.E. Mechanical Metallurgy, McGraw Hill Book Company, SI metric edition, London, p. 348, 1988.

2. Hertzberg, R.W. Deformation and fracture mechanics of engineering materials, John Wiley \& Sons, $4^{\text {th }}$ edition, New York , p. 315, 1996.

3. Ferreira, I. Tenacidade á fratura, em condições elastoplásticas, das ligas de alumínio de alta resistência do tipo Al-6Zn-2Mg-XCu, Faculdade de Engenharia Mecânica, Universidade Estadual de Campinas, Tese (Doutorado), 183 p., 1987.

4. American Society for Testing and Materials, E 39990, Standard Test Methods for Plane-Strain Fracture Toughness of Metallic Materials, Philadelphia, 31 p., 1995.

5. British Standard, 7448, Fracture mechanics toughness tests, Part 1, Method for determination of $\mathrm{K}_{\mathrm{IC}}$, critical CTOD and critical $\mathrm{J}$ values of metallic materials, 39 p., 1991.

6. Vareda, L.V. Avaliação das propriedades mecânicas de fadiga de baixo ciclo e alto ciclo e tenacidade à fratura de um aço inoxidavel austenítico do sistema Cr-Mn-N, Tese de Doutorado, Escola de Engenharia de São Carlos, USP, São Carlos - SP, Brazil, 233 p., 1997.

7. American Society for Testing and Materials, E 81389, Standard Test Methods for JIC, A Measure of Fracture Toughness, Philadelphia, 12 p., 1995.

8. Weels, A.A. Cranfield Crack Propagation Symposium, Unstable crack propagation in metals - Cleavage and fast fracture, p. 210, 1961.

9. Irwin, G.R. Analysis of stresses and strains near the end of a crack transversing a plate, Journal of Applied Mechanics, v. 24, 1957. In: Rolfe, S.T.; Barson, J.M. Fracture and Fatigue Control in Structures - Applications of Fracture Mechanics, Newmark, N.M.; Hall, W.J., eds., Prentice-Hall, New Jersey, p. 516, 1971.

10. Rolfe, S.T., Barson, J.M. Fracture and Fatigue Control in Structures - Applications of Fracture Mechanics, Newmark, N.M.; Hall, W.J., eds., Prentice-Hall, New Jersey, p. 516, 1971.

11. American Society for Testing and Materials, E 129093, Standard Test Methods for Crack-Tip Opening Displacement (CTOD) Fracture Toughness Measurement, Philadelphia, 10 p., 1995.

FAPESP helped in meeting the publication costs of this article 